\title{
EFECTO NEMATICIDA DE EXTRACTOS DE Quassia amara Y Brugmansia suaveolens SOBRE Meloidogyne sp. ASOCIADO AL TOMATE EN NICARAGUA ${ }^{1}$
}

\author{
Wilber Salazar-Antón², Tomás de Jesús Guzmán-Hernández ${ }^{3}$
}

\begin{abstract}
RESUMEN
Efecto nematicida de extractos de Quassia amara y Brugmansia suaveolens sobre Meloidogyne sp. asociado al tomate en Nicaragua. El objetivo de este trabajo fue evaluar extractos de hojas de Quassia amara L. (Simaroubaceae) y flores de Brugmansia suaveolens L. (Solanaceae) para determinar su efecto nematicida contra Meloidogyne sp. El estudio se realizó en el Campus Agropecuario de la Universidad Nacional Autónoma de Nicaragua-León (UNANLeón) en el año 2012. El material vegetal fue deshidratado, pulverizado y sus compuestos fueron extraídos utilizando como solvente metanol. Este estudio se realizó in vitro, se evaluó la mortalidad de los juveniles de Meloidogyne sp. en segundo instar después de 12, 24 y 48 horas de exposición a los extractos. Además, estos se aplicaron a plantas de tomate en maceteras bajo condiciones de invernadero, cuantificándose las poblaciones de nematodos a los 25, 50 y 75 días de exposición al extracto (DEE). En el experimento in vitro $Q$. amara y $B$. suaveolens diluidos al $10 \%$, presentaron los más altos porcentajes de mortalidad después de $48 \mathrm{~h}$, alcanzando 89 y $78 \%$ de juveniles muertos, respectivamente. En maceteras se evaluó la mortalidad de juveniles, índice de agallamiento (IA) y factor de reproducción (FR). Los mejores resultados para $Q$. amara se obtuvieron a los 25 DEE, con un porcentaje de mortalidad de $80 \%$, un IA de 1 en una escala de 0-5 y FR de 0,20. Con B. suaveolens, a los 25 DEE se alcanzó el mayor porcentaje de mortalidad de $71 \%$, un IA de 1,2 y un FR de 0,29. Estos resultados in vitro $\mathrm{y}$ en maceteras evidenciaron que ambos extractos poseen propiedades nematicidas, ya que redujeron significativamente $(\mathrm{P} \leq 0,05)$ las poblaciones de nematodos, su reproducción y el nivel de agallamiento de las raíces de tomate.
\end{abstract}

Palabras clave: cuasina, escopolamina, índice de agallamiento.

\begin{abstract}
Nematicidal effect of plant extracts from Quassia amara and Brugmansia suaveolens against Meloidogyne sp. on tomato plants in Nicaragua. The nematicidal activity of leaves of Quassia amara L. (Simaroubaceae) and flowers of Brugmansia suaveolens L. (Solanaceae) was evaluated against Meloidogyne sp. during a study conducted at the Agricultural Campus of the National Agricultural University in León (UNAN- León). The plant material was dehydrated, ground and the compounds quassin and scopolamine were extracted using methanol as a solvent. Then a study was conducted in vitro to evaluate mortality in second stage juveniles of Meloidogyne sp. after exposure to extracts (12, 24 and 48 hours). Subsequently, extracts were also evaluated in tomato plants established in pots under greenhouse conditions, and the corresponding nematode population was quantified after exposure to the extract $(25,50$ and 75 days of exposure to extract, DEE). In vitro tests with extracts of $B$. suaveolens and Q. amara diluted at $10 \%$ showed that the highest rate of nematode mortality occurs after $48 \mathrm{~h}$ of exposure, leaving $89 \%$ and $78 \%$ of all juveniles dead, respectively. In the test in pots, several aspects were evaluated including juvenile mortality, the plant galling index (GI), and the nematode reproduction factor (RF). The best result for $Q$. amara was obtained at $25 \mathrm{DEE}$, with $80 \%$ of mortality, a GI of 1 (in a scale from 0-5) and a RF of 0.2. Similarly, the best effect for B. suaveolens, was obtained at $25 \mathrm{DEE}$ with a $71 \%$ of mortality, a GI of 1.2 , and a RF of 0.29 . These results from tests in vitro and in pots results indicate that both extracts have nematicidal activities, as they significantly reduced the nematode population $(\mathrm{P} \leq 0.05)$, the reproductive ability and the incidence of root galling in tomato plants.
\end{abstract}

Palabras clave: quassin, scopolamine, galling index.

\footnotetext{
Recibido: 13 de junio, 2013. Aceptado: 18 de marzo, 2014. Este trabajo forma parte de la tesis de Doctorado en Ciencias Naturales para el Desarrollo (DOCINADE) del primer autor.

2 Laboratorio de Fitopatología, Departamento de Agroecología, Universidad Nacional Autónoma de Nicaragua-León. Nicaragua. Apartado postal N.64. Teléfono:(505) 311-1779. wilber.salazar@ct.unanleon.edu.ni

3 Instituto Tecnológico de Costa Rica, Sede San Carlos, Alajuela, Costa Rica. Apartado 159-7050, Florencia, Alajuela. Tel: (506) $2475-5310$. tjguzman@itcr.ac.cr
} 


\section{INTRODUCCIÓN}

El cultivo del tomate Solanum lycopersicum L. es uno de los vegetales de mayor consumo en Nicaragua debido a su sabor y alto contenido en vitaminas A, B y $\mathrm{C}$, adicionalmente provee una considerable cantidad de potasio, hierro y fósforo (Henriques da Silva et al., 2008). Una de las principales limitantes en su producción es el nematodo agallador (Meloidogyne spp.) (Salazar-Antón y Guzmán-Hernández, 2013), el cual causa pérdidas de alrededor de un $20 \%$ en la producción (Sasser y Freckman, 1987), y con un estimado de US \$ 500.00 millones invertidos para el control de este nematodo (Keren-Zur et al., 2000).

Meloidogyne spp. es del tipo endoparásito sedentario, al emerger del huevo, los juveniles invaden las raíces de sus hospederos e inducen la formación de células gigantes que utiliza para su alimentación (Hunt et al., 2005). Una vez que penetra las raíces del tomate, migra hacia el tejido vascular donde produce una serie de cambios fisiológicos y morfológicos que impiden a la planta la absorción de agua y nutrientes esenciales para su normal desarrollo (Castagnone-Sereno, 2006; Milligan et al., 1998).

En Nicaragua los nematodos fitoparásitos se combaten casi exclusivamente con nematicidas sintéticos, los cuales a pesar de ser efectivos y de rápida acción tienen las desventajas de contaminar fuentes de agua, ser altamente costosos y riesgosos para la salud humana (Wachira et al., 2009). Esto indica la importancia de la investigación en nematicidas botánicos, especialmente en la agricultura orgánica.

Existen unas 57 familias botánicas que contienen compuestos fitoquímicos con potencial para ser usados en el combate de enfermedades de plantas (Sukul, 1992). Entre estos se destacan aquellos con propiedades nematicidas como: terpenos, alcaloides, esteroides, taninos y aceites esenciales. Estos compuestos de origen vegetal tienen la ventaja de ser más seguros para el ambiente y la salud humana que sus contrapartes sintéticos y adicionalmente son considerados no persistentes en el campo (Chitwood, 2002).

Entre las plantas que han sido reportadas con propiedades nematicidas se encuentran Chamaesyce maculata, Euphorbia pulcherima y Lantana camara (Cox et al., 2006), Inula viscosa (Oka et al., 2006), Bixa Orellana (Oladoye et al., 2007), Tagetes erecta
$\mathrm{L}$, Hyptis suaveolens Poit y Ocimum gratissimum $\mathrm{L}$ (Olabiyi, 2008) y Ricinus communis (Arboleda et al., 2010).

$\mathrm{Al}$ menos treinta especies de plantas provenientes de catorce familias botánicas, se han validado con diferentes niveles de efectividad (Insunza et al., 2001). Esto demuestra la diversidad de esfuerzos que han sido realizados para encontrar moléculas naturales con efecto nematicida.

El objetivo de este trabajo fue evaluar extractos metanólicos de Quassia amara L. y Brugmansia suaveolens L., para determinar su efecto nematicida contra Meloidogyne sp. Estas plantas crecen naturalmente en Nicaragua $\mathrm{y}$ han sido reportadas con propiedades biocidas, insecticidas y nematicidas (Gutiérrez, 1988; Ocampo, 1994; López, 2010).

\section{MATERIALES Y MÉTODOS}

\section{Ubicación del estudio}

El proceso de extracción de los compuestos evaluados se realizó en el Laboratorio de Química del Instituto Tecnológico de Costa Rica, Sede San Carlos, Alajuela, Costa Rica. Las pruebas de mortalidad se realizaron en el Laboratorio de Fitopatología, ubicado en el Campus Agropecuario de la Universidad Nacional Autónoma de Nicaragua-León, Nicaragua, en el año 2012.

\section{Preparación del inóculo de Meloidogyne sp.}

Una masa de huevos obtenida de raíces agalladas de tomate Solanum lycopersicum var. Shanty susceptible a Meloidogyne sp. fue colocada en un beaker con agua destilada y un inyector de burbujas de aire por $24 \mathrm{~h}$, con el propósito de generar oxígeno para los nematodos. Los huevos y juveniles de segundo estadío fueron recuperados en tamices de 500 mesh y aplicados al suelo esterilizado colocado en maceteras que contenían una planta de tomate, previamente mantenidas en invernadero por 75 días (Castro et al., 2011). Posteriormente, se extrajo el sistema radical de las plantas anteriormente inoculadas y se obtuvieron huevos y juveniles de Meloidogyne sp., los cuales fueron liberados utilizando una solución al $0,5 \%$ de hipoclorito de sodio tal como fue descrito por Hussey y Janssen (2002). 


\section{Proceso de extracción de compuestos botánicos}

Las hojas de Quassia amara y flores de Brugmansia suaveolens fueron lavadas en agua potable, luego secadas y deshidratadas en un horno a $35^{\circ} \mathrm{C}$ por 96 horas y se pulverizaron en un molino foliar.

Los compuestos vegetales fueron extraídos de $350 \mathrm{~g}$ de material vegetal usando $200 \mathrm{ml}$ de metanol mediante un extractor Soxhlet por 24 horas. Posteriormente, la solución fue filtrada a través de un papel filtro Whatman No. 1 y el disolvente evaporado en un rotavapor por tres horas (Naz et al., 2012).

\section{Experimento in vitro}

Se evaluaron cinco concentraciones de cada extracto diluidos en agua destilada (1\% v/v, 2,5\% $\mathrm{v} / \mathrm{v}, 5 \% \mathrm{v} / \mathrm{v} 7,5 \% \mathrm{v} / \mathrm{v}$ y $10 \% \mathrm{v} / \mathrm{v})$, un testigo absoluto (agua destilada) y un testigo químico (oxamil 50 $\mathrm{mg} / \mathrm{l}$ ), se colocaron $20 \mathrm{ml}$ de estas soluciones en platos petri junto con 50 juveniles de segundo estadío de Meloidogyne sp. Los nematodos fueron colocados en cada plato e incubados por 12, 24 y 48 horas. Después de este tiempo, los nematodos fueron transferidos a un plato petri con agua destilada por 24 horas.

Se determinó la mortalidad de nematodos observándolos bajo un estereoscopio, aquellos que presentaban su cuerpo en posición recta y sin movimiento cuando se les tocaba con una aguja de disección, se consideraban muertos. Los datos se analizaron mediante un diseño completamente al azar $\left(\mathrm{Y}_{\mathrm{ij}}=\mu+\mathrm{t}_{\mathrm{i}}+\right.$ Eij) con siete tratamientos y cuatro repeticiones para un total de 28 unidades experimentales en cada uno de los tres periodos de incubación. Ambos experimentos fueron realizados dos veces.

\section{Experimento in planta}

Se utilizaron plantas de tomate var. Shanty susceptible a Meloidogyne sp., sembradas en un sustrato comercial estéril. A los veinte días de edad fueron trasplantadas a maceteras colocándose $1 \mathrm{~kg}$ de suelo, aplicándose $50 \mathrm{ml}$ de cada extracto a una concentración de 7,5\% v/v. Cinco días después, se aplicó una solución que contenía 200 juveniles en segundo estadío alrededor de la base del tallo de la plántula, adicionándose dos centímetros de suelo esterilizado para cubrir los nematodos (Hussey y Janssen, 2002). Las variables medidas fueron, porcentaje de mortalidad de
Meloidogyne sp., a los 25, 50 y 75 días de exposición al extracto (DEE). Se midió el índice de agallamiento (IA) a cada planta a los 75 DEE cuantificándose el número de agallas y asignándose un índice de acuerdo a la escala propuesta por Taylor y Sasser (1978). Esta sigue un esquema de $0-6$, donde $0=0$ agallas; $1=1-2$ agallas; $2=3-10$ agallas; $3=11-30$ agallas; $4=31-100$ agallas; $5=>100$ agallas.

La extracción de nematodos se realizó mediante el método de Baermann modificado (Hooper et al., 2005). Se pesaron $100 \mathrm{~g}$ de suelo y se colocaron en un filtro de papel toalla extendido sobre un tamiz dentro de un recipiente plástico. Luego se agregó agua hasta humedecer el nivel superior del suelo sin saturarlo. Se incubó por 48 horas y luego se filtró la suspensión dos veces a través de un tamiz de 400 mesh, colectando 20 $\mathrm{ml}$. Una vez obtenida la solución con los juveniles, se homogenizó para tomar 10 alicuotas de $5 \mathrm{ml}$ cada uno y cuantificar la población utilizando un estereoscopio (Castro et al., 2011).

Se midió el factor de reproducción (FR) a los 75 DEE, el cual es una medida de la capacidad reproductiva de los nematodos, utilizando la fórmula $\mathrm{R}=\mathrm{Pf} / \mathrm{Pi}$, donde $\mathrm{Pf}$ representa la población final de nematodos encontrados y Pi representa la población inicial de juveniles de segundo estadío que fueron inoculados.

Se utilizó un diseño completamente al azar $\left(\mathrm{Y}_{\mathrm{ij}}=\mu\right.$ $+t_{i}+$ Eij) en el que se evaluó el efecto de los extractos sobre Meloidogyne sp. a los 25, 50 y 75 DEE, un testigo absoluto (agua destilada) y un testigo químico (oxamil $50 \mathrm{mg} / \mathrm{l}$ ), cada uno de estos tratamientos se repitió cuatro veces, para un total de veinte unidades experimentales. Ambos experimentos fueron realizados dos veces. Posteriormente se realizó análisis de varianza y prueba de rango múltiple de Duncan al 0,5 cuando fue necesario.

\section{RESULTADOS Y DISCUSIÓN}

Efecto de cinco concentraciones de $Q$. amara y B. suaveolens sobre Meloidogyne sp. a nivel de laboratorio

Efecto de Quassia amara sobre nematodos in vitro

Las poblaciones de nematodos expuestas al extracto por 12, 24 y 48 horas, presentaron reducciones 
significativas $(\mathrm{P}<0,05)$ en todas las concentraciones evaluadas, en comparación con el testigo absoluto, lo que demuestra sus propiedades nematicidas. Los promedios de mortalidad más altos se lograron a las 48 horas en las diluciones al 7,5 y $10 \%$, donde se obtuvo un porcentaje de 88 y $89 \%$ de nematodos muertos respectivamente. Se comprobó que los factores, tiempo y concentración del extracto fueron fundamentales en la mortalidad de nematodos (Cuadro 1). La viabilidad de los juveniles decreció en la medida que se utilizaba una dilución más alta (10\%) por un periodo de tiempo más largo (48 h). El testigo absoluto (agua destilada) presentó el nivel más bajo de mortalidad con $13 \%$, siendo superada por todos los tratamientos evaluados. El testigo químico (oxamil) presentó el nivel más alto de mortalidad con $96 \%$ (Cuadro 1).

Niveles de mortalidad similares a los encontrados en este estudio fueron reportados previamente por López (2010), quien al evaluar la mortalidad in vitro de Radopholus similis observó un $86 \%$ de mortalidad utilizando extractos de $Q$. amara. Estos altos niveles de mortalidad han sido atribuidos por Coats (1994) a metabolitos secundarios presentes como aminoácidos y alcaloides. De este último, se destaca la cantina que posee propiedades citotóxicas, que causan daño a las células de organismos vivos (López y Pérez, 2008).

Por otro lado, Mancebo et al. (2000) reportan la cuasina, como un alcaloide que posee potencial nematicida y que igualmente está presente en $Q$. amara. La cuasina es un terpeno descrito como el compuesto más activo que utiliza la planta en contra de organismos que la atacan, lo que le permite ejercer una acción biocida sobre organismos fitoparásitos (Ocampo, 1994; López, 2010).

\section{Efecto de B. suaveolens sobre nematodos in vitro}

Se comprobó que las mayores mortalidades de juveniles de segundo estadío de Meloidogyne sp. fueron encontradas a las 48 horas de exposición en las diluciones al 7,5 y $10 \%$ con promedios de 77 y $78 \%$ respectivamente, resultados similares a los del experimento anterior. Estos porcentajes fueron significativamente $(\mathrm{P}<0,05)$ más altos que los porcentajes de mortalidad encontrados en los otros tratamientos y claramente superiores a los porcentajes de mortalidad hallados en el tratamiento testigo (agua destilada). El testigo químico, con promedios de juveniles muertos de 43 (12 horas), 44 ( 24 horas) y 46 (48 horas), presentó los niveles más altos de mortalidad de juveniles de Meloidogyne sp. con porcentajes de entre 86 al 92\% (Cuadro 2).

Estudio previos demostraron que la mortalidad causada por B. suaveolens sobre Meloidogyne sp. se debe a la presencia de alcaloides del grupo tropano, como escopolamina, hiosciamina y atropina (Kvist y Moraes, 2006). En particular la escopolamina es ampliamente conocida por poseer propiedades nematicidas, responsables directas de la mortalidad encontrada en las evaluaciones realizadas en el presente experimento (Shahwar et al., 1995).

El porcentaje de mortalidad de nematodos más alto fue de $78 \%$, lo que representa un alto efecto nematicida, sin embargo, estos resultados son inferiores

Cuadro 1. Porcentaje de mortalidad de juveniles de segundo estadío Meloidogyne sp. expuestos al extracto de Q. amara en condiciones in vitro en el año 2012. León, Nicaragua.

\begin{tabular}{lccc}
\hline & \multicolumn{2}{c}{ Tiempo de exposición de juveniles a extractos de Q. amara } \\
\cline { 2 - 4 } & $\mathbf{1 2}$ horas & $\mathbf{2 4}$ horas & $\mathbf{4 8}$ horas \\
\hline Testigo absoluto & $4,88 \pm 0,83 \mathrm{a}$ & $6,50 \pm 1,51 \mathrm{a}$ & $6,62 \pm 2,20 \mathrm{a}$ \\
Dilución al 1.0\% & $14,25 \pm 2,82 \mathrm{~b}$ & $16,13 \pm 2,80 \mathrm{~b}$ & $17,75 \pm 4,50 \mathrm{~b}$ \\
Dilución al 2.5\% & $24,00 \pm 3,46 \mathrm{c}$ & $24,25 \pm 2,82 \mathrm{c}$ & $25,75 \pm 3,77 \mathrm{c}$ \\
Dilución al 5.0\% & $28,37 \pm 2,26 \mathrm{~d}$ & $28,88 \pm 4,22 \mathrm{~d}$ & $30,12 \pm 4,52 \mathrm{~d}$ \\
Dilución al 7.5\% & $40,25 \pm 3,65 \mathrm{e}$ & $43,62 \pm 3,74 \mathrm{e}$ & $44,00 \pm 2,98 \mathrm{e}$ \\
Dilución al 10.0\% & $40,00 \pm 3,25 \mathrm{e}$ & $43,25 \pm 3,85 \mathrm{e}$ & $44,50 \pm 3,89 \mathrm{e}$ \\
Testigo químico & $42,38 \pm 4,41 \mathrm{f}$ & $47,12 \pm 1,36 \mathrm{f}$ & $48,37 \pm 1,19 \mathrm{f}$ \\
\hline
\end{tabular}

Números seguidos por la misma letra y dentro de la misma columna no son significativamente diferentes $(\mathrm{P} \leq 0,05)$ según la prueba de Rangos múltiples de Duncan al 5\%. 
Cuadro 2. Porcentaje de mortalidad de juveniles de Meloidogyne sp. expuestos al extracto de B. suaveolens en condiciones in vitro en el año 2012. León, Nicaragua.

\begin{tabular}{lccr}
\hline & \multicolumn{2}{c}{ Tiempo de exposición de juveniles a extractos de B. suaveolens } \\
\cline { 2 - 4 } & $\mathbf{1 2}$ horas & 24 horas & 48 horas \\
\hline Testigo absoluto & $6,75 \pm 2,55 \mathrm{a}$ & $6,87 \pm 1,64 \mathrm{a}$ & $7,25 \pm 1,28 \mathrm{a}$ \\
Dilución al 1,0\% & $11,25 \pm 2,66 \mathrm{~b}$ & $14,00 \pm 3,63 \mathrm{~b}$ & $15,37 \pm 2,92 \mathrm{~b}$ \\
Dilución al 2,5\% & $21,00 \pm 2,93 \mathrm{c}$ & $22,37 \pm 3,58 \mathrm{c}$ & $24,75 \pm 2,60 \mathrm{c}$ \\
Dilución al 5,0\% & $28,87 \pm 2,80 \mathrm{~d}$ & $30,62 \pm 4,37 \mathrm{~d}$ & $30,87 \pm 4,32 \mathrm{~d}$ \\
Dilución al 7,5\% & $33,62 \pm 3,62 \mathrm{e}$ & $38,00 \pm 4,44 \mathrm{e}$ & $38,75 \pm 4,50 \mathrm{e}$ \\
Dilución al 10,0\% & $34,75 \pm 4,27 \mathrm{e}$ & $38,62 \pm 3,66 \mathrm{e}$ & $39,00 \pm 4,69 \mathrm{e}$ \\
Testigo químico & $43,00 \pm 4,07 \mathrm{f}$ & $44,25 \pm 4,86 \mathrm{f}$ & $46,25 \pm 4,95 \mathrm{f}$ \\
\hline
\end{tabular}

Números seguidos por la misma letra y dentro de la misma columna no son significativamente diferentes $(\mathrm{P} \leq 0,05)$ según la prueba de Rangos múltiples de Duncan al 5\%.

a los obtenidos por Gutiérrez (1988) quien encontró niveles de mortalidad de hasta $91 \%$ en juveniles de Meloidogyne sp. Posteriormente, Insunza et al. (2001) realizó nuevas evaluaciones con escopolamina, y encontró que este ejerció una alta actividad tóxica contra fitonematodos. Estos autores evaluaron las propiedades nematicidas in vitro de escopolamina contra el nematodo fitoparásito Xiphinema americanum el cual luego de ser expuesto al extracto por 24 horas presentó promedios de mortalidad que oscilaron entre 88 y $100 \%$ de mortalidad.

Efecto de $Q$. amara y $B$. suaveolens sobre Meloidogyne sp. asociado a tomate en maceteras

Efecto de Quassia amara sobre Meloidogyne sp. en maceteras

En los muestreos de nematodos realizados a los 25, 50 y 75 días de exposición al extracto (DEE), se observó que las poblaciones de Meloidogyne sp. fueron reducidas significativamente en comparación con el testigo sin aplicación de extractos. Los mayores niveles de eficacia del extracto fueron encontrados a los 25 DEE, encontrándose únicamente cuarenta juveniles vivos representando un $80 \%$ de mortalidad de un total de 200 juveniles inoculados (Cuadro 3). Al cuantificar el IA se obtuvo un índice de 1,1 en la escala de 0-5 sugerida por Taylor y Sasser (1978), lo que representa de una a dos agallas por planta (Figura 1). Adicionalmente, al evaluar el FR se obtuvo un factor de 0,2 , el cual por ser menor que 1 indica que la población del nematodo en lugar de crecer, se redujo, en este caso en un $80 \%$, coincidiendo con el bajo nivel de daño reportado en el IA. Esto indica que las poblaciones de Meloidogyne sp. vieron reducida su capacidad de reproducirse e infectar a la planta en presencia del extracto.

Las evaluaciones hechas a los 50 y 75 DEE demostraron una elevación tanto en los niveles poblacionales, como en el IA de la planta y en el FR de Meloidogyne sp. A los 50 DEE el porcentaje de mortalidad fue reducido a $65 \%$ cuantificándose setenta nematodos vivos, el IA sufrió un leve aumento y fue medido en 1,62 y el FR alcanzó a 0,34. Las mediciones a los 75 DEE demostraron un incremento en los niveles poblacionales de juveniles de Meloidogyne sp. al reducirse la mortalidad de estos a 46,37\%, representando 107 nematodos vivos. Igualmente se aumentaron el IA a 1,87 y el FR a 0,53 (Figura 2).

Estos resultados señalan que el efecto nematicida del extracto de $Q$. amara decrece con el tiempo. Esto podría deberse a la degradación natural del extracto al exponerse a las condiciones del ambiente, lo cual reduce su efectividad con el paso del tiempo. Esta degradación de los principios activos del extracto de Q. amara fue previamente reportado por Flores et al. (2008) quienes encontraron que los compuestos que causan la inhibición alimenticia se descomponen y pierden su efecto al exponerse a las condiciones ambientales. Algunos alcaloides encontrados en los extractos de $Q$. amara, como es el caso de los cuasinoides pueden ser descompuestos por la luz y probablemente por la exposición al aire (Flores et al., 2008). 
Cuadro 3. Efecto de extractos de Q. amara y B. suaveolens sobre la sobrevivencia y el factor de reproducción de juveniles de Meloidogyne sp. en condiciones in vitro en el año 2012. León, Nicaragua.

\begin{tabular}{lcccc}
\hline \multirow{2}{*}{ Tratamiento } & \multicolumn{2}{c}{ Extracto de $Q$. amara } & \multicolumn{2}{c}{ Extracto de $\boldsymbol{B}$. suaveolens } \\
\cline { 2 - 5 } & Sobrevivencia & Factor de reproducción & Sobrevivencia & Factor de reproducción \\
\hline Testigo químico & $29,26 \pm 9,14 \mathrm{a}$ & $0,14 \pm 0,05 \mathrm{a}$ & $30,00 \pm 5,02 \mathrm{a}$ & $0,15 \pm 0,03 \mathrm{a}$ \\
Extracción 25DEE & $40,76 \pm 11,08 \mathrm{~b}$ & $0,20 \pm 0,03 \mathrm{~b}$ & $58,00 \pm 6,60 \mathrm{~b}$ & $0,29 \pm 0,09 \mathrm{~b}$ \\
Extracción 50DEE & $69,76 \pm 9,74 \mathrm{c}$ & $0,34 \pm 0,06 \mathrm{c}$ & $79,74 \pm 9,82 \mathrm{c}$ & $0,39 \pm 0,07 \mathrm{c}$ \\
Extracción 75DEE & $107,26 \pm 11,36 \mathrm{~d}$ & $0,53 \pm 0,06 \mathrm{~d}$ & $109,50 \pm 13,04 \mathrm{~d}$ & $0,54 \pm 0,08 \mathrm{~d}$ \\
Testigo absoluto & $244,50 \pm 19,12 \mathrm{e}$ & $1,22 \pm 0,10 \mathrm{e}$ & $249,24 \pm 18,24 \mathrm{e}$ & $1,24 \pm 0,09 \mathrm{e}$ \\
\hline
\end{tabular}

Números seguidos por la misma letra y dentro de la misma columna no son significativamente diferentes $(\mathrm{P} \leq 0,05)$ según la prueba de rangos múltiples de Duncan al 5\%.

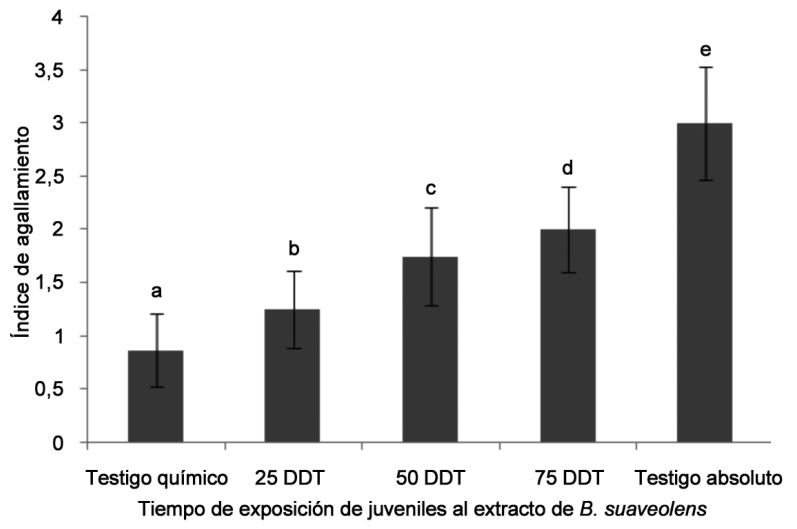

Figura 1. Índice de agallamiento ocasionado por juveniles de Meloidogyne sp. en raíces de tomate expuestos a extractos de Q. amara en el año 2012. León, Nicaragua.

La mortalidad de nematodos expuestos a extractos de $Q$. amara fue reportada previamente por López (2010) y podría ser explicada por la presencia de compuestos como cuasina y neocuasina en dichos extractos (López y Pérez, 2008). Estos alcaloides inhiben la capacidad de alimentación de diferentes organismos fitófagos (Mancebo et al., 2000). Sobre este tema Hilje y Stansly (2001), encontraron propiedades disuasivas alimenticias en estos dos cuasinoides. Por otro lado, Veierov (1996) afirma que estos alcaloides reducen los niveles de energía y causan deshidratación a los organismos expuestos a ellos.

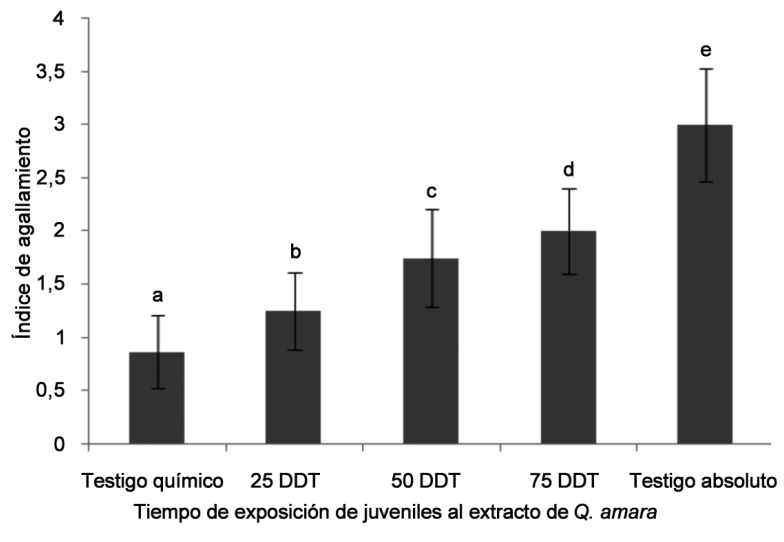

Figura 2. Índice de agallamiento ocasionado por juveniles de Meloidogyne sp. en raíces de tomate expuestos a extractos de B. suaveolens en el año 2012. León, Nicaragua.

El IA en este estudio fue en promedio menor o igual a 2, esto podría deberse a las propiedades de las cuasinas y neocuasinas las que han demostrado ser capaces de inhibir la alimentación de organismos plagas como los insectos (Hilje y Stansly, 2001). Niveles de agallamiento menores a dos son considerados bajos, ya que de acuerdo con Canto-Saénz (1983), son más tolerantes, lo que sugiere que el extracto reduce el IA a niveles que son tolerables por la planta.

El factor de reproducción (FR) de Meloidogyne sp. se redujo con las aplicaciones de extractos de $Q$. amara, observándose el mayor efecto en el tratamiento 
evaluado a los 25 días de exposición al extracto con un FR de 0,20. Otros autores encontraron FR de 9 al evaluar variedades de tomate categorizadas como muy susceptibles a Meloidogyne sp. con suelo esterilizado y sin ningún método de control (Navarro-Barthelemy et al., 2009). Sin embargo, Castro et al. (2011), al utilizar vermicompost y quitina lograron reducir el FR en tomate susceptible a 3,76 versus 0,20 a 0,53 en el presente estudio, lo que hace suponer el potencial que posee este extracto como un nematicida alternativo.

El FR encontrado, brinda información muy importante sobre la utilidad del extracto debido a que es un indicador de la eficiencia del hospedero (Jiménez, 1985), demostrando que plantas a las que se les aplica el extracto se vuelven hospedero menos receptivos para las poblaciones de nematodos.

\section{Efecto de B. suaveolens sobre nematodos en maceteras}

Las evaluaciones realizadas a los 25,50 y 75 DEE indican que las poblaciones de juveniles de Meloidogyne sp. en los tratamientos evaluados fueron reducidas por efecto del extracto en comparación con el testigo. Los mayores niveles de control fueron observados a los 25 DEE, encontrándose 58 nematodos vivos, lo que representa una mortalidad del $71 \%$ del total de 200 juveniles inoculados (Cuadro 3). El IA ocasionado por los nematodos fue de 1,25 , equivalente a una o dos agallas por planta siendo insuficiente para causar un daño severo a la planta (Figura 2). Por otro lado, el FR fue de 0,29 indicando que las poblaciones decrecieron en presencia del extracto, reduciéndose su capacidad de reproducirse e infectar las plantas.

Las evaluaciones realizadas a los 50 y 75 DEE demostraron un incremento en los niveles poblacionales de Meloidogyne sp., así como una elevación en los IA y FR. A los 50 DEE el porcentaje de mortalidad del nematodo se redujo a $60 \%$, demostrando un incremento poblacional encontrándose 80 nematodos vivos (Cuadro 3), el IA sufrió un leve incremento y fue medido en 1,7 y el FR fue medido en 0,39. Las mediciones a los 75 DEE muestran un $45 \%$ de mortalidad, lo que demuestra un aumento en la población de Meloidogyne sp. a 110 nematodos vivos, el IA se elevó a 2,0 y el FR también llegando a 0,54 . El incremento en estas dos últimas variables en comparación con las evaluaciones hechas a los 25
DEE muestra que los nematodos tuvieron en las dos últimas evaluaciones menos dificultades para penetrar la planta, infectarla y reproducirse.

La mortalidad causada por extractos de $B$. suaveolens sobre Meloidogyne sp. puede ser atribuida a alcaloides tóxicos que producen plantas de la familia solanácea como la atropina, hioscina y escopolamina (Pino y Alvis, 2008; Pino, 2010). De estos alcaloides, se sabe que la escopolamina es el alcaloide que se presenta en mayores concentraciones en $B$. suaveolens (Flores-Hernández, 2011) y el que posee mayor potencial nematicida (Shahwar et al., 1995).

De igual manera, Gutiérrez (1988) utilizó extractos de Brugmansia sp. y encontró un 91\% de mortalidad en poblaciones de Meloidogyne sp. asociados a tomate en maceteras. Posteriormente, otros autores evaluaron nuevamente su actividad nematicida y encontraron niveles de mortalidad entre 90 y $100 \%$ sobre Hoplolaimus indicus, Helicotylenchus multicinctus, y M. incognita (Hussain et al., 2011). Los niveles de agallamiento en tomate, fueron reducidos gradualmente en la medida que se incrementaba la dosis de escopolamina, donde se demostró una correlación inversa entre las concentraciones de este alcaloide aplicado al suelo y el nivel de infestación de Meloidogyne sp. (Abid, 1996).

En el presente estudio el IA promedio en los tratamientos con extracto de B. suaveolens fue de 1,6 , lo que demuestra la efectividad del extracto como nematicida ya que un índice menor a 2 indica que los nematodos presentaron limitaciones en su capacidad de dañar las plantas (Jiménez, 1985). Esto podría ser atribuido a las propiedades repelentes y disuasivas alimenticias que le han sido atribuidas a este extracto (Pérez y Iannacone, 2008). El IA menor que 2 obtenido, es propio de plantas tolerantes a Meloidogyne sp. según la escala de resistencia propuesta por Canto-Sáenz (1983). El estudio reporta que el IA del tratamiento testigo fue de 3, lo que indica que la variedad utilizada es susceptible según los parámetros utilizados por el mismo autor (CantoSáenz, 1983). De lo anterior, se deduce que los bajos IA obtenidos en las plantas tratadas con el extracto fueron debido al efecto protector de este y no a la tolerancia de la variedad utilizada.

El FR en los tratamientos con extractos no superó 0,54 en las poblaciones de Meloidogyne sp. lo que indica que los nematodos no incrementaron 
en presencia del extracto, sino que por el contrario, decrecieron. Este FR menor a 1 es propio de plantas tolerantes según la escala propuesta por Suarez y Rosales (2008). En este caso, el bajo FR no se debe a la tolerancia de la variedad utilizada, ya que el tratamiento testigo absoluto tuvo un FR de 1,24, sino a la acción protectante del extracto observado por la tolerancia de las plantas de tomate a Meloidogyne sp.

\section{LITERATURA CITADA}

Abid,M. 1996. Studies on the control of Root-knot nematodes (Meloidogyne spp.) with botanical toxicants. Department of Botany, National Nematological Research Centre, University of Karachi.

Arboleda, F.J., O.A. Guzmán Piedrahita, y J.F. Restrepo Henao. 2010. Efecto in vitro de extractos acuosos de higuerilla (Ricinus communis Linneo) sobre el nematodo barrenador [Radopholus similis (Cobb) Thorne]. Agronomia 18(2):25-36.

Canto-Saénz, M. 1983. The nature of resistance to Meloidogyene incognita (Kofoid \& White 1919) Chitwood 1949. En: Carter, C.C., editor, Proc. Third Res. \& Plann.Conf. on Root knot nematodes Meloidogyne spp. International Meloidogyne Project, Lima, Peru. p. 160-165.

Castagnone-Sereno, P. 2006. Genetic variability and adaptative evolution in parthenogenetic root-knot nematodes. Heredity 96:282-289.

Castro,L.,L.Flores,y L.Uribe. 2011.Efectodel vermicompost y quitina sobre el control de Meloidogyne incognita en tomate a nivel de invernadero. Agronomía Costarricense 35(2):21-32.

Chitwood, D.J. 2002. Phytochemical based strategies for nematode control. Annual Review of Phytopathology 40:221-9.

Coats, J. 1994. Risks from natural versus synthetic insecticides. Annual Revision Entomology EEUU 39:489-515

Cox, C.J., L.B. McCarty, J.E. Toler, S.A. Lewis, y S.B. Martin. 2006. Suppressing sting nematodes with Brassica sp., Poinsettia and spotted spurge extracts. Agron. J. 98: 962-967.

Flores-Hernández, M.A. 2011. Compilación bibliográfica Brugmansia spp. Facultad de Ciencias Químicas, Universidad Veracruzana, México. p. 1-49.

Flores, G., L. Hilje, G.A. Mora, y M. Carballo. 2008 Antifeedant activity of botanical crude extracts and their

ISSN: $2215-3608$ fractions on Bemisia tabaci (Homoptera: Aleyrodidae) adults: III. Quassia amara (Simaroubaceae). Rev. Biol. Trop. 56(4):2131-2146.

Gutiérrez, P. 1988. Uso de extractos vegetales para el control de nematodos en el cultivo del tomate (Lycopersicon esculentum Miller). Tesis Licenciatura en Ciencias Agrícolas. Facultad de Agronomía, Universidad de San Carlos, Costa Rica.

Henriques da Silva, D.J., F. Barbosa, F.R. Brunele, A. Castro, y V. Patel. 2008. Tomatoes: origin, cultivation techniques and germplasm resources. En: V. Preddy, y R. Watson, editores, Tomatoes and tomato products: nutritional, medicinal and therapeutic properties. Science Publisher, Enfield, NH, USA. p. 3-25.

Hilje, L., y P.A. Stansly. 2001. Development of crop associations for managing geminiviruses vectored by whiteflies in tomatoes. Final report. U.S. Dept. Agr. (USDA). CATIE, Turrialba, Costa Rica.

Hooper, D.J., J. Hallmann, y S.A. Subbotin. 2005. Methods for extraction, processing and detection of plant and soil nematodes. En: M. Luc, R.A. Sikora, y J. Bridge, editores, Plant parasitic nematodes in subtropical and tropical agriculture. 2 ed, CABI, UK. p. 53-86.

Hunt, D., Luc, M; Manzanilla-López, R. 2005. Identification, morphology and biology of plant parasitic nematodes En: M. Luc, R.A. Sikora, y J. Bridge, editores, Plant parasitic nematodes in subtropical and tropical agriculture. CABI publishing. p. 11-52.

Hussain, M.A., T. Mukhtar, y M.Z. Kayani. 2011. Efficacy evaluation of Azadirachta indica, Calotropis procera, Datura stramonium and Tagetes erecta against rootknot nematodes Meloidogyne incognita. Pak. J. Bot., 43:197-204.

Hussey, R.S., y G. Janssen. 2002. Root-knot nematodes: Meloidogyne species. En: J.L. Starr, R. Cook, y J. Bridge, editores, Plant resistance to parasitic nematodes. USA. CAB International. p. 43-70.

Insunza, V., E. Aballay, y J. Macaya. 2001. In vitro nematicidal activity of aqueous plant extracts on Chilean populations of Xiphinema americanum sensu lato. Nematropica 31:47-54.

Jiménez, M. 1985. Evaluación de resistencia de cinco cultivares de tomate (Lycopersicon esculentum Mill) al ataque de Meloidogyne spp. IDESIA (Chile) 9:15-20.

Keren-Zur, M., J. Antonov, A. Bercovitz, A. Feldman, G. Keram, N. Morov, y N. Rebhum. 2000. Baccillus firmus formulation for the safe control of root knot nematodes. En: The BCPC Conference: Pests and disease. Brighton, United Kingdom. p. 307-311.

AGRONOMÍA MESOAMERICANA 25(1):111-119. 2014 
Kvist, L.P., y R.M. Moraes. 2006. Plantas sicoactivas. Botánica Económica de los Andes Centrales 12:294-312.

López, J.A., y J. Pérez. 2008. Etnofarmacología y actividad biológica de Quassia amara (Simaroubaceae): Estado de la cuestión. Boletín Latinoamericano y del Caribe de Plantas Medicinales 7(5):234-246.

López, A.P. 2010. Evaluación de extractos de cuatro especies de plantas y sus compuestos orgánicos sobre la mortalidad de Radopholus similis en condiciones in vitro. Tesis Lic. en Ingeniería en Agronomía, Instituto Tecnológico de Costa Rica, sede San Carlos, Alajuela, Costa Rica.

Mancebo, F., L. Hilje, G.A. Mora, y R. Salazar. 2000. Antifeedant activity of Quassia amara (Simaroubaceae) extracts on Hypsipyla grandella (Lepidoptera: Pyralidae) larvae. Crop Prot. 19:301-305.

Milligan, S., J. Bodeau, J. Yaghoobi, I. Kaloshian, P. Zabel, y V.M. Williamson. 1998. The root knot nematode resistance gene Mi from tomato is a member of the leucine zipper, nucleotide binding, leucine-rich repeat family of plant genes. Plant Cell 10:1307-1319.

Navarro-Barthelemy, L., L. Gómez, R. Enrique, F.M. González, y M.G. Rodríguez. 2009. Comportamiento de genotipos de tomate (Solanum lycopersicum L) frente a Meloidogyne incognita. Rev. Protección Veg. 24(1):54-56.

Naz, I., J.E. Palomares-Rius, Saifullah, V. Blok, M.R. Khan, y S. Ali. 2012. In vitro and in planta nematicidal activity of Fumaria parviflora against the southern root-knot nematode Meloidogyne incognita. Plant Pathology 62(4):943-952.

Ocampo, R. 1994. Estudios etno-botánico de las palmas empleadas por los indígenas en Talamanca, Costa Rica. Revista Forestal (CR) 7:1-6.

Oka, Y., B.H. Ben-Daniel, y Y. Cohen. 2006. Control of Meloidogyne javanica by formulations of Inula viscosa Leaf extracts. J. Nematol. 38(1):46-51.

Olabiyi, T.I. 2008. Pathogenicity study and nematoxic properties of some plant extracts on root knot nematode pest of tomato, Lycopersicon esculentum (L.) Mill. Plant Pathology Journal 7(1):45-49.

Oladoye, S.O., T.I. Olabiyi, E.T. Ayodele, y G.T. Ibikunle. 2007. Photo-Chemical screening and nematicidal potential of root bark extract of Bixa orellana on nematode pests. Res. Crops 8:222-228.

Pérez, D., y J. Iannacone. 2008. Mortalidad y repelencia en Eupalamides cyparissias (Lepidoptera:Castniidae), plaga de la palma aceitera Elaeis guineensis, por efecto de diez extractos botánicos. Rev. Soc. Entomol. Argent. 67(1-2):41-48.

Pino, V.E. 2010. Efecto de extractos vegetales en la reducción poblacional de Meloidogyne sp. Ingeniera Agropecuaria. Tesis Ing. Agropecuaria, Fac. de Ciencias Agrop., Universidad Técnica de Babahoyo, Ecuador.

Pino J., y R. Alvis. 2008. Efecto de Brugmansia arborea (L.) Lagerheim (Solanacea) en el sistema reproductor masculino de ratón. Rev Per Biol. 15(2):125-128.

Salazar-Antón, W., y T.J. Guzmán-Hernández. 2013. Efecto de poblaciones de Meloidogyne sp. en el desarrollo y rendimiento del tomate. Agron. Mesoam. 24(2):419426.

Sasser, J.N., y D.W. Freckman. 1987. A world perspective on Nematology: the role of the society. En: V.A. Veech, y D.W. Dickson, editores, Vistas on nematology. Society of Nematologists, Hyattsville, Maryland. p. 7-14.

Shahwar, D., M. Abid, A.U. Rehman, M.A. Maqbool, y M.I. Choudhary. 1995. Nematicidal compounds from Datura fastuosa. En: M.A. Atta-ur-Rehman, M.S. Choudhary, y H.E.J. Sheikhani, editores, Proc. $19^{\text {th }}$ IUPAC Symposium on the chemistry of Natural Products. Res. Inst. of Chemistry, University of Karachi, Karachi 75270- Pakistan. p. 171-179.

Suarez, H.Z., y L.C. Rosales. 2008. Comportamiento de materiales genéticos de piña (Ananas comosus) al ataque de Meloidogyne incognita raza 1. Rev. Protección Veg. 23(3):191-195.

Sukul, N.C. 1992. Plants antagonistic to plant-parasitic nematodes. Indian Review of Life Sciences 12:23-52.

Taylor, A.L., y J.N. Sasser. 1978. Biology, identification and control of some root-knot nematodes (Meloidogyne spp.). A coop. Pub. Dept. Plant Pathology and Genetics, North Carolina State University y USAID. Raleigh, N.C.

Veierov, D. 1996. Physically and behaviorally active formulations for control of Bemisia. En: D. Gerling, y R.T. Mayer, editores, Bemisia 1995: Taxonomy, biology, damage, control and management. Intercept, Andover, United Kingdom. p. 557-576.

Wachira, P.M., J.W. Kimenju, S.A. Okoth, y R.K. Mibey. 2009. Stimulation of nematode-destroying fungi by organic amendments applied in management of plant parasitic nematode. Asian J. Plant. Sci. 8(2):153159. 